\title{
A SILICON OLFACTORY BULB OSCILLATOR
}

\author{
Vitor M. Grade Tavares, Jose C. Principe, John G. Harris \\ University of Florida \\ Dept. of Electrical and Computer Engineering \\ 451 Engineering Building, PO Box 116130, Gainesville, FL 32611-6130, U.S.A.
}

\begin{abstract}
This paper presents a low power MOS-VLSI implementation of an oscillator proposed by Freeman to model a very important component of the olfactory cortex [1]. The model dynamics has time constants in the order of $1 / 220$ (s). To accomplish the long time constants a new filtering technique recently proposed [2][3] was utilized. All the blocks involving information processing were designed to operate below threshold (weak inversion)
\end{abstract}

\section{INTRODUCTION}

Freeman has been developing since 1975 a biologically realistic computational model of the mammalian olfactory cortex [1]. Up to now the system has been simulated with Runge-Kutta numerical methods [4]. The method is excellent to find numerical solutions of differential equations, however in some circumstances, such as the case of an on-line system, it may not be the best choice for computational reasons. We have recently proposed the use of DSP (Digital Signal Processing) techniques [5] as an alternative to find the system solutions. The method will not be as accurate as Runge-Kutta, specially if the sampling frequency is not very high, however in dynamical terms the solutions are identical with similar frequency response and observable behavior. More importantly, the impact of the technique to the VLSI implementation side is substantial. The DSP approach immediately sets a synchronous time for the events. Each output point is updated every $T$ seconds (where $\mathrm{T}$ is the sampling period), which means that control over time is possible allowing transparent multiplexing procedures. It spares resources without the need for extra filtering as it would be the case if applied to a continuous time analog implementation[6]. Based on this work, this paper proposes a VLSI implementation with sampled time and analog magnitudes. With the approach we still retain the inherent parallelism of an analog computation with consequent compactness and infinite magnitude resolution (no quantization problems), but at the same time the sampling procedure gives more flexibility to the design allowing multiplexing wuthout extra burdens. The circuits described here were designed and fabricated with MOSIS $1.2 \mu$ technology.

\section{THE KII MODEL: A QUICK OVERVIEW}

The KII model fits in a hierarchy of structures made up of basic processing elements (PEs) designated $\mathrm{K} 0$ (figure 1) that -can be either inhibitory or excitactory. Figure 1 shows an example of an excitactory $\mathrm{K} 0$, the inhibitory is simply obtained by multiplying the output of figure 1 by negative one (flipping the sigmoidal nonlinearity over the $x$ axis)

These PEs are modular and formal representations of populations of neurons in the mammalian brain. In this sense the model does not intend to represent individual responses of a neuron but a set of neurons as a whole. The formal representation of responses are done by means of density functions that will measure the firing pulse density of a set of neurons and conversely the density of pulse to wave conversion. Hence, the functions are continuous in time, magnitude and space. From biological evidence Freeman as proposed the following $\mathrm{K} 0$ structure [1] shown in figure 1 to represent a population of neurons.

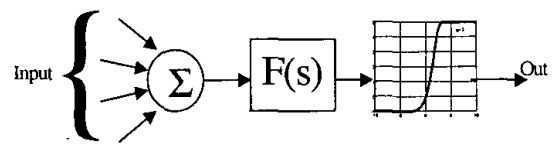

Figure 1.- K0 model diagram (excitactory).

K0 comprises a summing input node where the spacial inputs converge into a $2^{\text {nd }}$ order dynamics block $F(s)$ represented by (1). The output signal is the result of a pulse to wave density conversion function. This signal is then reconverted into a pulse density signal by means of a non-symmetric non-linear function represented also in figure 1. This is the wave to pulse conversion phase and, in terms of densities, is functionally represented by (2). Figure 2 shows the non-linearity for different ' $q$ ' values.

$$
\begin{gathered}
\frac{1}{a \cdot b}\left(\frac{\partial^{2}}{\partial t^{2}} x(t)+(a+b) \frac{\partial}{\partial t} x(t)+a \cdot b x(t)\right)=f(t) \\
Q(x(t), q)= \begin{cases}q\left(1-e^{-\left(e^{x(t)}-1\right) / q}\right) & \text {, If } x(t)>x_{0} \\
-1 & , \text { If } x(t)<x_{0}\end{cases} \\
x_{0}=\ln (1-q \ln (1+1 / q))
\end{gathered}
$$

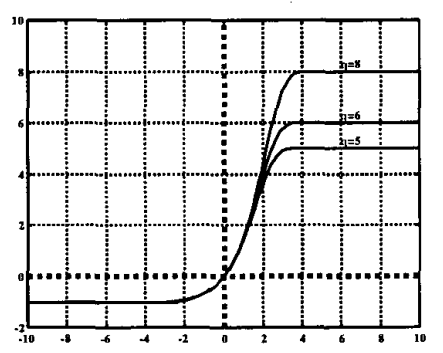

Figure 2.- Non-symmetric non-linearity function (eq. (2)). 


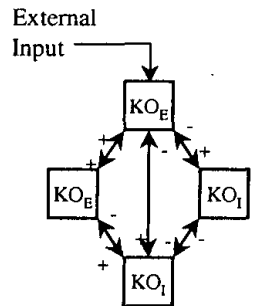

Figure 3.- KII model representation (each square represents a $\mathrm{K} 0$ of figure 1 , the indexes I and $\mathrm{E}$ stand for inhibitory and excitatory, respectively).

The KII is a special arrangement of K0s. The arrangement falls from biological evidence and is shown in figure 3 . Under correct parameter set, defined as interconnection gains, the KII model behaves as an oscillator controlled by the input. It is zero for zero input and oscillates if the input (an external signal shown in figure $3)$ amplitude rises. The (+) and (-) signs describe excitactory and inhibitory connections.

\section{THE NON-SYMMETRIC NON-LINEAR CIRCUIT IMPLEMENTATION}

The K0 sigmoidal function has a prominent exponential behavior as equation (2) and figure 2 attests. A precise MOS-VLSI implementation of (2) requires a lot of components. However in the subthreshold region an approximation can be very easily attained with a modified OTA (Operational Transconductor Amplifier). Working below threshold will further save power consumption, which is one of the goals of this project. The most noticeable feature of equation (2) is the unbalanced saturation levels. The plot of this function shows more displacement towards the positive (excitation) than the negative (inhibition) side (figure 2).

Figure 4 presents the circuit schematic that gives the necessary sigmoidal behavior. The non-linear function is characterized by a wide-range differential stage amplifier (non-linear cell). The necessary biasing current is accomplished by unbalancing a current mirror. This procedure obviously introduces big off-sets, however these can be compensated by adding a reference voltage from a similar cell as the figure shows (off-set compensator cell). Finally the $\mathrm{G}_{\mathrm{m} 3}$ transconductor acts as a voltage to current converter. The source degenerated diodes were included to enlarge the linear operation area in relation to the wide range OTA making this solely responsible for the non-linear shape.

The DC cut-off limits are then given by $\mathrm{nI}_{\mathrm{b}-\mathrm{NL}}$ (related with $\mathrm{q}$ in (2) and figure 2 ) for positive inputs and $\mathrm{I}_{\mathrm{b}-\mathrm{NL}}$ for the negative. This means that although the saturation limits can be programmed with the biasing current, its relative displacement (ratio of upper saturation level versus the lower) is constant and given by the transistors mismatch ' $n$ ' shown in figure 4 . The result measured from the chip is shown in figure 5 for two different biasing currents.

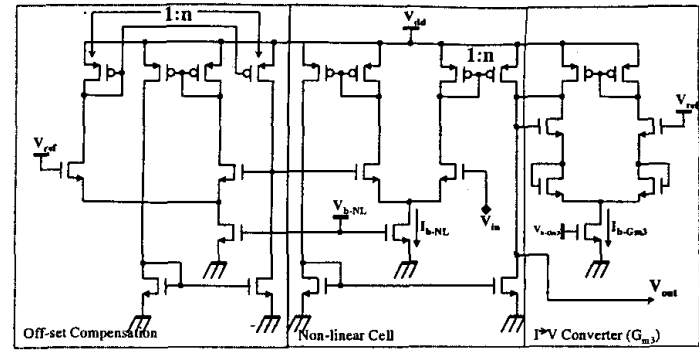

Figure 4.- Implemented Non-symmetric Non-linear function (voltage mode)

\section{F\&H IMPLEMENTATION OF THE DYNAMICS}

The KO model is characterized by second order dynamics as it was shown in section 2. The differential equation is linear and corresponds to a $2^{\text {nd }}$ order low-pass filter with poles approximately at $35 \mathrm{~Hz}$ and $114 \mathrm{~Hz}$. At such low frequencies either SC (switched capacitor) [7] or SI (switched current) [8] need high capacitor or transistor ratios respectively. Furthermore, in general they are not low power implementations for reasons of slew-rate and linearity. In order to meet the low frequency, low area and low power requirements, a new filtering technique called Filter and Hold $(\mathrm{F} \& \mathrm{H})$ was proposed [2][3].

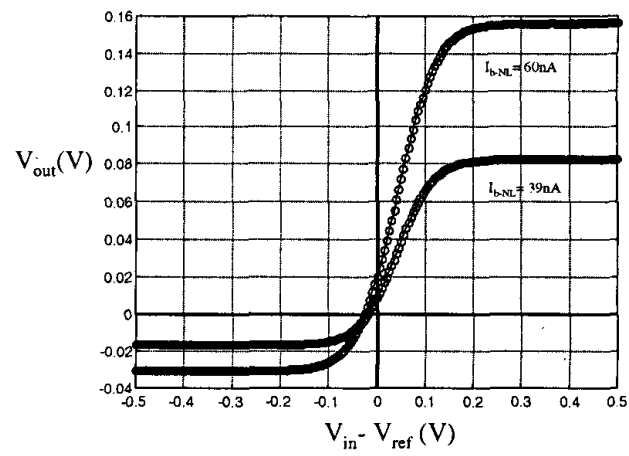

Figure 5.- Non-linear DC response measured from the non-linear circuit shown in figure 5 (the bias current of $\mathrm{G}_{\mathrm{m} 3}$ is $\mathrm{I}_{\mathrm{b}-\mathrm{Gm} 3}=600 \mathrm{nA}$ ).

The F\&H concept magnifies a time constant by down scaling a given time constant $\mathrm{RC}$ through a periodically gating process of a capacitor charge with a given duty-cycle $\mathrm{k}$ (the scaling factor). Figure 6 shows the F\&H utilized for the KO dynamics. 

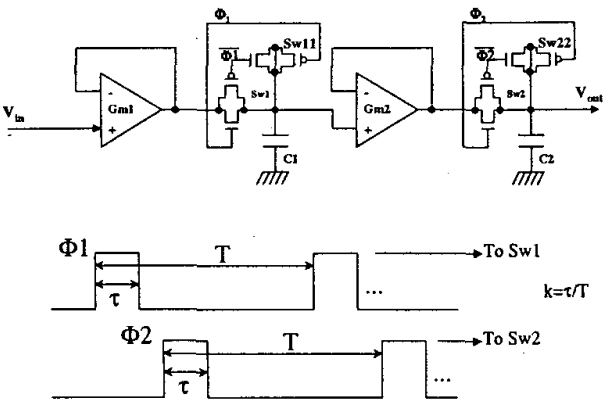

Figure 6.- Implemented $\mathrm{F} \& \mathrm{H}$ approximation of the differential equation (1)

Originally the continuous time filter was calculated for frequencies 100 times higher $(3.5 \mathrm{kHz}$ and $11.4 \mathrm{kHz}$ ), the down scaling is then accomplished by clocking the switches $S_{\mathrm{w} 1}$ and $S_{\mathrm{w} 2}$ with a duty-cycle clock of $k=1 \%$ (the sampling frequency was chosen to be 20 times bigger than the highest pole in the filter). The $G_{m}$ amplifier is topologically the same as $\mathrm{G}_{\mathrm{m} 3}$ in figure 4 . The source degenerated diodes increase the input dynamic range in relation to the non-linearity, and it also decreases the $G_{m}$ value which further decreases the necessary capacitor values. Figure 7 shows the measured results (the biasing current of the $\mathrm{G}_{\mathrm{m}}$ amplifiers is $10 \mathrm{nA}$ ). The very low frequency values are in this way accomplished with a very low power implementation $(100 n \mathrm{~W})$ and with a fairly low area consumption of $330 \mu m \times 250 \mu m\left(C_{1}=1 \mathrm{pF}, \mathrm{C}_{2}=4 \mathrm{pF}\right)$.

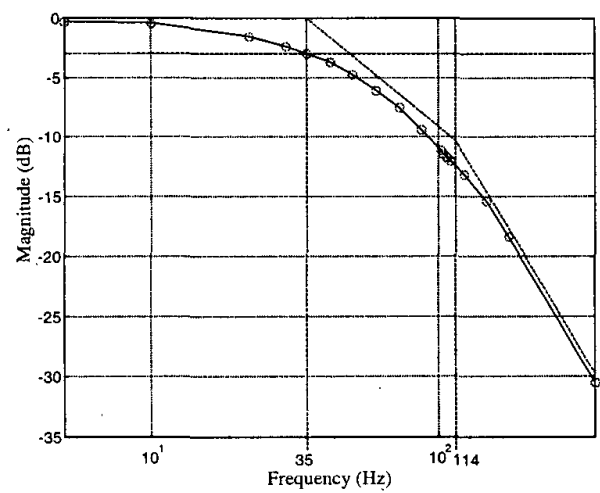

Figure 7.- F\&H Measured $\mathrm{AC}$ response.

\section{THE KO INHIBITORY AND EXCITATORY CELLS.}

A complete the $\mathrm{K} 0$ model requires a summing block besides the dynamics and non-linearity. Figure 8 shows the K0 with the summing block schematic.

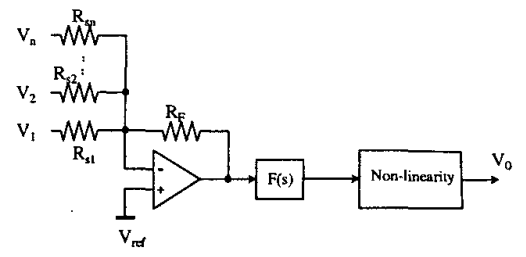

Figure 8.- K0 model with a voltage summing node.

The voltage adder configuration inverts the input signals. In order to have the proper signal sign between input and output, the nonlinear circuits were topologically changed to reverse the signal polarity. Figure 9 shows conceptually the needed non-linear function change to ensure the proper signs.

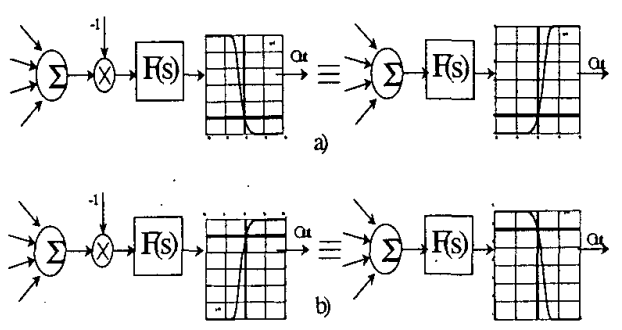

Figure 9.- Block diagram of the implemented $\mathrm{K} 0$, a) Excitactory $\left.\left(\mathrm{KO}_{\mathrm{E}}\right), \mathrm{b}\right)$ - Inhibitory $\left(\mathrm{KO}_{\mathrm{I}}\right)$.

\section{KII MEASURED RESULTS}

The KII setup is shown in figure 10 , with table I summarizing the parameter values. The results from an actual VLSI implementation are plotted in figures 11 and 12 . As expected the KII behaves as an oscillator controlled by the input. The waveform phases measured at different points of the KII follow closely the digital simulations.

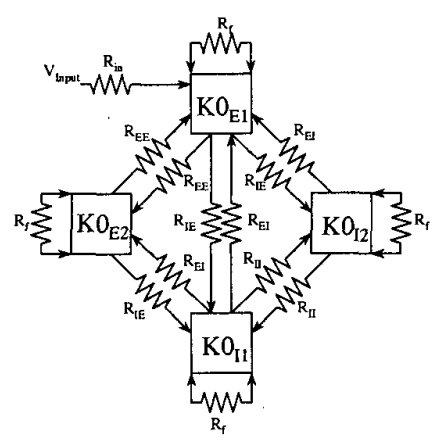

Figure 10.- KII schematic $\left(\mathrm{R}_{\mathrm{F}}\right.$ and $\mathrm{R}_{\mathrm{ij}}$; $\mathrm{j}, \mathrm{i}=\mathrm{E}$ or $\mathrm{I}$, correspond to $\mathrm{R}_{\mathrm{sk}} ; \mathrm{k}=1, \ldots, \mathrm{n}$ in figure 7 , respectively). 


\section{CONCLUSION}

Table 1: KII Interconnections gain

\begin{tabular}{|l|c|}
\hline \multicolumn{1}{|c|}{ Gain } & Value \\
\hline \hline $\mathbf{R}_{\mathrm{F}} / \mathbf{R}_{\mathrm{EE}}$ & 2.5 \\
\hline $\mathbf{R}_{\mathrm{F}} / \mathbf{R}_{\mathbf{I I}}$ & 2.5 \\
\hline $\mathbf{R}_{\mathrm{F}} / \mathbf{R}_{\mathbf{I E}}$ & 2 \\
\hline $\mathbf{R}_{\mathbf{F}} / \mathbf{R}_{\mathbf{E I}}$ & 5 \\
\hline
\end{tabular}

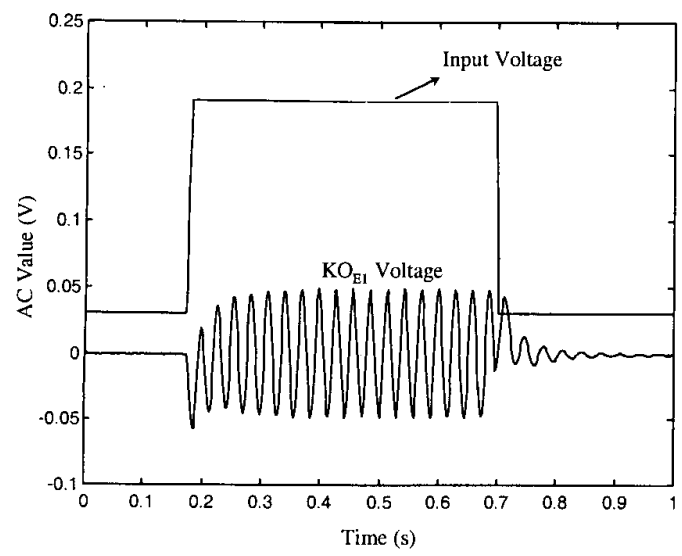

Figure 11.- Measured KII oscillatory behavior with the input.

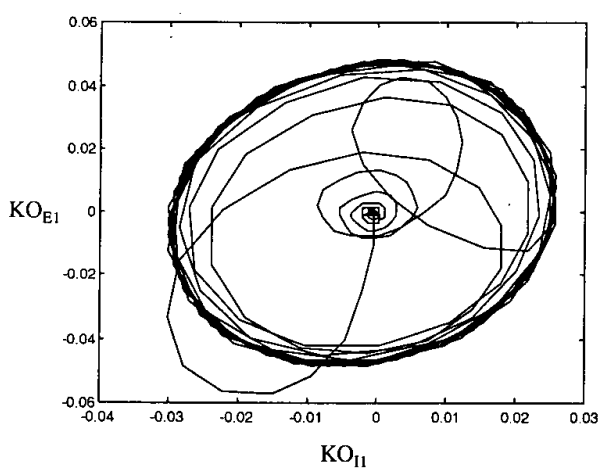

Figure 12.-Measured KII phase plot showing a complete cycle correspondent to figure 11 . All K0 cells in figure 10 oscillate at the same frequency but out of phase.
This paper presents the first mixed mode, sub-threshold, VLSI implementation of an oscillator that models the basic processing element of the olfactory bulb, an important piece of the olfactory system in mammals. The results are in agreement with the theory and conclusions already reported [5][9]. The initial DSP proposal to digitize the system was fundamental to a practical and successful VLSI implementation. When the goal is the implementation of a single KII model a discrete time implementation may seem questionable. However our goal here is to build a chip with an array of ' $n$ ' coupled KIIs, which means $n^{2}$ interconnection gains among them. In this case the discrete time design enables time multiplexing which may be the only way to conduct the design. Moreover, discrete time also facilitates the implementation of long time constants with the $\mathrm{F} \& \mathrm{H}$ solution.

\section{ACKNOWLEDGMENTS}

To FCT (Fundacao para a Ciencia e Tecnologia) in Portugal for sponsoring Vitor Tavares.

\section{REFERENCES}

[1] W. Freeman "Mass action in the nervous system", Academic Press, 1975.

[2] V. M. G. Tavares, J. C. Principe and J. G. Harris "F\&H Filter: A Novel ultra-low power discrete time filter", Elect. Letters, Vol. 35 No 15, July 1999

[3] V. M. G. Tavares, J. C. Principe, J. G. Harris, and P. G. Oliveira, "Filter and hold circuit utilizing a charge/discharge current", U.S. Provisional Patent Application, August 1999

[4] W. H. Press, Et al "Numerical Recipes: The Art of Scientific Computing", Cambridge Univ. Press., Cambridge (England), 1996.

[5] V. Tavares, J. Principe, and G. Lynn "Discrete network implementation of Freeman's model of the olfactory system", Proc. IEEE WCNN-IJNN, 1998

[6] J. Eisenberg, W. Freeman, B Burke "Hardware architecture of a neural network model simulating pattern recognition by the olfactory bulb" Neural Networks, 2, 1989.

[7] R. W. Brodersen, P. Gray, and, D. Hodges "MOS SwitchedCapacitor Filters" Proc. IEEE, vol. 67, Jan. 1979

[8] J. Hughes, N. Bird, and I. Macbeth "Switched currents - A new technique for analog sampled-data signal processing", International Symposium on Circuits and Systems, 1989.

[9] Y. Yao, W. J. Freeman, and B. Burk "Central Pattern Generating and Recognizing in Olfactory Bulb: A Correlation Learning Rule", Neural Networks, Pergamon Press, Vol. 1, 1988. 